\title{
Le rôle des acteurs régionaux dans l'attraction et la rétention des travailleurs en région périphérique : des activités aux couleurs économiques et sociales
}

\author{
Andrée-Anne Deschênes ${ }^{\mathrm{a}}$, Catherine Beaudry ${ }^{\mathrm{b}}$ et Josée Laflamme ${ }^{\mathrm{c}}$
}

\section{INTRODUCTION}

Les organismes de développement régional

sont alors appelés à intervenir pour attirer et retenir les travailleurs, et ce, afin de

poursuivre et de consolider le développement économique et social de la région.

Une des principales préoccupations des dirigeants québécois réside dans la capacité de leur entreprise à attirer et à retenir une main-d'œuvre qualifiée. Cette préoccupation est d'autant plus perceptible dans les régions périphériques du Québec, où les facteurs démographiques liés au vieillissement de la population et à l'exode des jeunes vers les grands centres accroissent les difficultés des entreprises à pourvoir leur besoin en main-d'œuvre. En région éloignée, les pratiques mises en place par les employeurs seuls ne suffisent pas pour faire face à cet enjeu crucial. Les organismes de développement régional sont alors appelés à intervenir pour attirer et retenir les travailleurs, et ce, afin de poursuivre et de consolider le développement économique et social de la région. Leur soutien est d'autant plus nécessaire que la plupart des entreprises situées en région périphérique, souvent de petite ou de moyenne taille (PME), peinent à rivaliser avec les grandes entreprises en matière d'attributs organisationnels attractifs et fidélisants.

À l'issue d'une étude portant sur les interventions des acteurs régionaux dans la région du Bas-SaintLaurent (située dans l'est du Québec), cet article propose de dresser un portrait des activités déployées sur le plan de l'attraction et de la rétention des travailleurs dans la région. Alors que la plupart des recherches s'intéressent aux facteurs organisationnels qui favorisent l'attraction et la rétention des travailleurs, comme les conditions et l'environnement de travail, rares sont celles qui se penchent sur les facteurs liés au milieu de vie et sur les interventions externes à l'entreprise.

\section{LE RÔLE DES ACTEURS RÉGIONAUX : ATTIRER ET RETENIR AU-DELÀ DES FACTEURS ORGANISATIONNELS}

Parmi les facteurs régionaux favorisant

l'attraction et la rétention des travailleurs se trouvent l'état du marché du travail et la croissance économique d'une région'. Dans cet ordre d'idées, des opportunités de carrière intéressante dans une région contribuent à sa capacité à attirer et retenir des travailleurs.

D'un point de vue pratique et scientifique, l'attraction et la rétention des travailleurs en entreprise sont généralement appréhendées en se limitant à des considérations organisationnelles. Or, la mise en lumière des facteurs régionaux de l'attraction et de la rétention est essentielle, puisqu'en région périphérique particulièrement, la satisfaction à l'égard du travail ne suffit pas. La région doit également combler les attentes et les besoins des travailleurs, de manière à ce qu'ils décident de s'installer dans une région et qu'ils ne migrent pas vers d'autres régions.

Parmi les facteurs régionaux favorisant l'attraction et la rétention des travailleurs se trouvent l'état du marché du travail et la croissance économique d'une région ${ }^{1}$. Dans cet ordre d'idées, des opportunités de carrière intéressante dans une région contribuent à sa capacité à attirer et retenir des travailleurs ${ }^{2}$ L'environnement économique d'une région est donc considéré comme un facteur d'importance et il peut être évalué par : le marché du travail, le nombre d'entreprises (ou les possibilités de carrières), la croissance économique et les domaines d'activités économiques ${ }^{3}$. 
Qui plus est, la culture régionale et la qualité de vie ne sont pas sans conséquence sur la capacité d'attraction et de rétention régionales. La culture régionale réfère aux politiques de gestion régionale (par exemple celles liées à l'urbanisme ou celles soutenant l'innovation) et au dynamisme entrepreneurial, alors que la qualité de vie repose sur le marché hypothécaire et locatif, les installations sportives et récréatives, l'offre culturelle et les politiques visant le soutien à l'établissement des familles ${ }^{4}$. L'accessibilité à une qualité de vie qui favorise l'intégration et le développement d'un sentiment d'appartenance envers une région constitue un levier majeur pour y attirer et y retenir des travailleurs talentueux.

Les employeurs peuvent certes promouvoir ces atouts régionaux et contribuer à leur développement. Toutefois, leurs actions prises isolément ne suffisent pas à attirer et à retenir les travailleurs qui sont de plus en plus sollicités. L'étude des activités

\section{MÉTHODOLOGIE}

Le questionnaire de recherche a été construit par une équipe d'universitaires familiarisés avec les concepts d'attraction et de rétention régionale. Il comptait 49 questions, dont cinq sur le profil des organisations sondées, 23 sur l'attraction, 14 sur la rétention, deux sur l'impact perçu et cinq sur l'image de la région. Les questions, sauf celles portant sur les caractéristiques de l'organisme, soumettent un choix de réponse mesuré sur une échelle de Likert en 5 points, allant de « pas du tout en accord » à « tout à fait en accord ». Celles-ci visent à connaître les activités, en matière d'attraction et de rétention des travailleurs des acteurs régionaux pour attirer et retenir la maind'œuvre trouve alors tout son sens, alors que les entreprises situées en région périphérique dépendent aussi des facteurs régionaux pour atteindre leurs objectifs stratégiques. Comme la clé de la croissance régionale réside entre autres dans la concentration d'un noyau de travailleurs productifs ${ }^{5}$, les différents organismes de développement bénéficient eux aussi de la présence accrue de travailleurs. L'objectif de cet article est donc de mettre en lumière les actions déployées en ce sens par les acteurs régionaux de la région du Bas-Saint-Laurent.

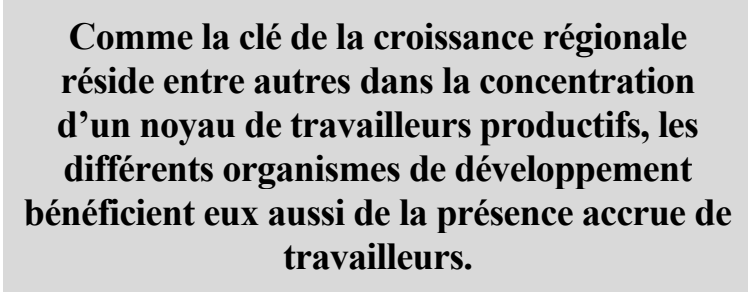

dans la région, déployées par les organismes au sein desquels œuvrent les répondants. Les données ont été recueillies à l'été 2013 par voie électronique. Sur les 218 sollicités, 77 acteurs régionaux des huit Municipalités régionales de comté (MRC) du territoire bas-laurentien ont répondu au questionnaire. Ceux-ci proviennent de divers organismes dont la mission est liée au développement régional. À ce titre, le tableau 1 présente le domaine d'activités, le statut et les territoires couverts par les organismes sondés ainsi que les postes occupés par les répondants.

Tableau 1 - Portrait de l'échantillon

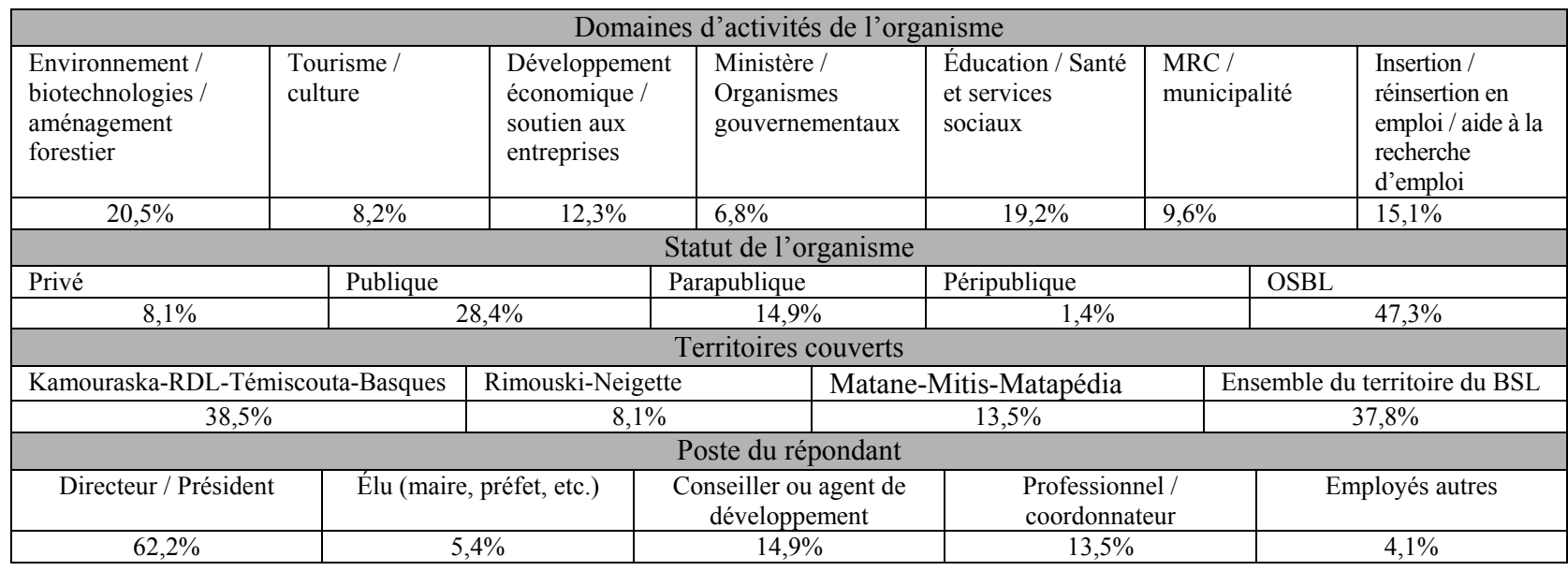


Pour compléter cette enquête par questionnaire, deux groupes de discussion ont été menés à l'automne 2013. Ces groupes ont respectivement rassemblé huit et cinq participants et ont duré deux heures chacun. Ils regroupaient à la fois des acteurs régionaux et des employeurs du BasSaint-Laurent. Les données recueillies par le biais

\section{RÉSULTATS}

\subsection{Attirer : la promotion des caractéristiques régionales à l'extérieur de la région}

La majorité des organismes interrogés comprend des activités relevant d'un volet lié à l'attraction de travailleurs dans la région $(53,8 \%)$. Principalement, les activités visant l'attraction des travailleurs dans la région concernent la promo- de ces entrevues ont été retranscrites en verbatim et codifiées selon les thèmes à l'étude. L'objectif de ces groupes de discussion était de discuter de la pertinence des pratiques actuelles et futures des acteurs régionaux relativement aux deux enjeux que sont la rétention et l'attraction de travailleurs en région.

\section{Tableau 2 - Caractéristiques régionales promues à l'extérieur de la région}

\begin{tabular}{|cll|} 
Rang & Caractéristiques régionales & Moyenne \\
\hline 1 & Le dynamisme entrepreneurial & 3,08 \\
2 & Les domaines d'activités économiques & 3,04 \\
3 & L'état du marché du travail & 2,95 \\
4 & L'offre culturelle & 2,83 \\
5 & La croissance économique & 2,81 \\
6 & Les installations sportives et récréatives & 2,78 \\
7 & Les politiques de gestion régionales & 2,75 \\
8 & Les politiques familiales & 2,71 \\
9 & Le nombre d'entreprises & 2,49 \\
10 & Le marché hypothécaire et locatif & 2,05 \\
\hline
\end{tabular}

L'importance accordée aux caractéristiques économiques est notable; le dynamisme entrepreneurial, les domaines d'activités économiques et l'état du marché du travail se hissent en tête de peloton. En ce qui a trait à la promotion de la qualité de vie, l'offre culturelle prime sur les installations sportives et récréatives, les politiques familiales et le marché hypothécaire et locatif, qui closent le palmarès.

Une autre activité déployée par les acteurs régionaux en vue d'attirer des travailleurs est la promotion d'une image positive de la région. En effet, une forte majorité de répondants $(79,5 \%)$ ont indiqué que leur organisme travaille à promouvoir une image positive du Bas-Saint-Laurent. À cet égard, une majorité de répondants $(55,1 \%)$ considèrent que les résidents du Bas-Saint-Laurent tion des caractéristiques régionales auprès de la population des autres régions. Ces caractéristiques régionales sont associées à l'environnement économique, à la qualité de vie et à la culture régionale. Le tableau 2 présente les caractéristiques régionales promues à l'extérieur de la région, selon l'importance des activités mises en œuvre par les acteurs régionaux. 
présenter le Bas-Saint-Laurent comme milieu de vie, car pour que les travailleurs choisissent cette région plutôt qu'une autre, ils doivent impérativement connaître les possibilités d'y travailler. Malgré l'importance de promouvoir l'emploi, les participants mentionnent que les attraits de la région relatifs à la qualité de vie et aux divertissements doivent aussi être valorisés. En effet, selon eux, les activités liées à l'attraction seront efficaces si elles permettent aux travailleurs d'imaginer le Bas-SaintLaurent comme milieu de vie, où l'épanouissement personnel et les défis professionnels sont possibles. Par ailleurs, les activités culturelles, les installations sportives et récréatives, la beauté des paysages de même que les services éducatifs, communautaires ou de santé doivent être présentés.

Le discours des participants aux groupes de discussion et les résultats de l'étude quantitative se rejoignent en ce qui a trait à l'importance de la promotion des facteurs économiques, plus particulièrement ceux liés à l'emploi. Toutefois, une différence importante est présente lorsqu'il est question de la promotion des attraits liés à la qualité de vie. Il y a donc un écart entre ce qui est fait par les acteurs régionaux (les résultats au questionnaire) et ce qui devrait être fait selon eux (les résultats aux groupes de discussion).

Les participants sont divisés au regard de la promotion de ces caractéristiques régionales en fonction des différents territoires. Certains misent sur une promotion globale de la région baslaurentienne, alors que d'autres sont d'avis que les caractéristiques propres à chaque MRC devraient être présentées. Ils soulignent entre autres les disparités au regard des besoins de main-d'œuvre et de la capacité attractive entre les milieux en bordure du littoral et ceux situés dans les terres, plus ruraux.

En outre, selon les participants, la promotion de ces attraits devrait viser des catégories particulières de population. Ils insistent sur le fait que les stratégies d'attraction mises en œuvre par les acteurs régionaux ciblent essentiellement les jeunes de 35 ans et moins. Ils estiment que les jeunes familles ont davantage d'intérêt et de facilité à migrer que celles dont les enfants sont devenus adolescents. C'est dans cette perspective qu'ils indiquent l'importance de viser plus spécifiquement les travailleurs âgés de 35 à 45 ans, personnes qui détiennent déjà une expérience de travail. Les participants sont également d'avis que la promotion auprès des touristes porterait ses fruits, car ces derniers ont déjà un intérêt pour la région.

Par ailleurs, les participants aux groupes de discussions ont fait ressortir que la sensibilisation des employeurs au problème d'attraction régionale constitue un point de départ incontournable. Ils estiment que les directions d'entreprise, surtout celles des PME, ne réalisent pas l'ampleur de l'éventuel problème.

\subsection{Retenir : des actions orientées vers l'environnement économique d'abord}

La sensibilisation des employeurs au problème d'attraction régionale constitue un point de départ incontournable. Ils estiment que les directions d'entreprise, surtout celles des PME, ne réalisent pas l'ampleur de l'éventuel problème.

Plus de la moitié (59\%) des organismes sondés déploient des activités en lien avec la rétention des travailleurs dans la région. Plus précisément, les répondants ont été questionnés sur la contribution de leur organisme, par le biais de leurs programmes et pratiques, quant à la rétention des travailleurs au Bas-Saint-Laurent. Les résultats, présentés au tableau 3, révèlent que les activités des acteurs régionaux s'orientent davantage vers les questions d'ordre économique. En effet, les organismes travaillent dans une plus grande proportion à consolider les domaines d'activités, à diminuer le taux de chômage, à diversifier le marché du travail et les domaines d'activités économiques et à soutenir les entrepreneurs. L'attraction et la rétention de nouvelles entreprises, bien que situées au sixième et septième rang du palmarès, font l'objet de moins d'interventions de la part des acteurs régionaux que les autres des enjeux économiques. Au regard de la culture régionale, la création de politiques de gestion régionale semble moins présente. Les organismes déploient moins d'efforts en ce qui concerne la qualité de vie. Leurs activités se concentrent principalement sur la création de politiques familiales et sur l'offre culturelle de la région. Viennent en tout dernier lieu les activités liées au développement d'installations sportives et récréatives, à l'accessibilité aux logements et à l'aide à la recherche de logement. 
Tableau 3 - Activités des organisations liées à la rétention

\begin{tabular}{|cll|}
\hline Rang & Activités de l'organisme & Moyenne \\
\hline 1 & Consolider les domaines d'activités & 3,83 \\
2 & Diminuer le taux de chômage & 3,74 \\
3 & Diversifier le marché du travail & 3,69 \\
4 & Diversifier les domaines d'activités & 3,60 \\
5 & Soutenir les entrepreneurs & 3,56 \\
6 & Retenir les entreprises dans la région & 3,36 \\
7 & Attirer des entreprises & 3,13 \\
8 & Créer des politiques familiales & 3,13 \\
9 & Créer des politiques de gestion régionales & 3,13 \\
10 & Augmenter l'offre culturelle & 3,01 \\
11 & Développer des installations sportives et & 2,64 \\
& récréatives & \\
12 & Développer l'accessibilité aux logements & 2,39 \\
13 & Aider à la recherche de logement & 2,31 \\
\hline
\end{tabular}

Afin de retenir les travailleurs dans la région, les acteurs régionaux cherchent également à valoriser certaines caractéristiques régionales auprès de la population bas-laurentienne. Ces dernières sont présentées au tableau 4 en fonction de l'ordre d'importance que les acteurs régionaux y accordent au regard de leur promotion.

Tableau 4 - Caractéristiques régionales valorisées dans la région

\begin{tabular}{|cll|}
\hline Rang & Caractéristiques régionales & Moyenne \\
\hline 1 & Domaines d'activités économiques & 3,47 \\
2 & L'état du marché du travail & 3,42 \\
3 & Le dynamisme entrepreneurial & 3,32 \\
4 & La croissance économique & 3,18 \\
5 & Les politiques familiales & 3,08 \\
6 & L'offre culturelle & 3,01 \\
7 & Les politiques de gestion régionales & 2,97 \\
8 & Le nombre d'entreprises & 2,87 \\
9 & Les installations sportives et & 2,82 \\
& récréatives & \\
10 & Le marché hypothécaire et locatif & 2,01 \\
\hline
\end{tabular}

Les caractéristiques économiques, soit les domaines d'activités, l'état du marché du travail, le dynamisme entrepreneurial et la croissance économique, sont celles étant le plus souvent mises en valeur.

Les caractéristiques économiques, soit les domaines d'activités, l'état du marché du travail, le dynamisme entrepreneurial et la croissance économique, sont celles étant le plus souvent mises en valeur. Viennent ensuite les politiques familiales et l'offre culturelle, qui sont liées à la qualité de vie dans la région. Les politiques de gestion régionale et le nombre d'entreprises sont des caractéristiques moins promues. Finalement, les installations sportives et récréatives et le marché hypothécaire et locatif ferment la liste.

En groupe de discussion, les participants expliquent l'importance de déployer des efforts supplémentaires pour favoriser la rétention de travailleurs au Bas-Saint-Laurent. À l'instar des enjeux d'attraction, ils mettent en lumière la 
nécessité de sensibiliser les employeurs aux défis de la rétention régionale. Ils considèrent qu'à l'heure actuelle, une majorité d'employeurs ne saisit pas l'importance du problème.

Par ailleurs, les participants expliquent que la mise en valeur des caractéristiques économiques, notamment celles liées aux emplois disponibles et recherchés dans la région, devrait davantage se faire auprès des jeunes bas-laurentiens, et ce, dès le secondaire. C'est notamment de cette manière qu'il sera possible de freiner leur exode vers d'autres régions du Québec. De plus, les participants mentionnent l'importance de la collaboration entre les employeurs et entre les acteurs régionaux, notamment en ce qui concerne l'aide à la recherche d'emploi pour les conjoints des nouveaux arrivants et pour l'organisation d'activités sociales. Finalement, le développement d'une structure officielle d'accueil et d'intégration pour les travailleurs non originaires de la région serait un incontournable.

Les résultats du sondage signifient clairement que les activités sont axées essentiellement sur l'économie, alors que les participants mettent l'accent sur le développement des facteurs plus sociaux de la rétention.

Au même titre que pour l'attraction régionale, le discours des participants aux groupes de discussion et les résultats issus du questionnaire présentent des différences. Les résultats du sondage signifient clairement que les activités sont axées essentiellement sur l'économie, alors que les participants mettent l'accent sur le dévelop-

\section{DISCUSSION ET CONCLUSION}

La majorité des acteurs régionaux sondés contribue directement ou indirectement à la création et au maintien des emplois existants. Ce constat est positif en matière d'attraction et de rétention de la maind'œuvre, car les perspectives professionnelles pour les travailleurs découlent directement de la disponibilité des emplois. pement des facteurs plus sociaux de la rétention. Ces résultats révèlent les disparités entre les activités déployées par les acteurs régionaux et celles qui devraient l'être, selon les participants.

\subsection{Faire la différence : les acteurs régionaux considèrent-ils que leurs activités ont un impact?}

Lorsque les acteurs régionaux sont interrogés sur leur perception des impacts qu'ont les activités qu'ils déploient en ce qui a trait à l'attraction des travailleurs dans la région, la majorité d'entre eux est très en accord $(41 \%)$ ou tout à fait en accord $(17,9 \%)$ avec le fait que leurs activités font une différence. Ce sont ceux œuvrant dans les organismes dont la mission est liée à l'insertion en emploi et à la recherche d'emploi qui considèrent que leurs activités ont le plus d'impact sur l'attraction de la main-d'œuvre dans la région. Dans une moindre proportion $(17,9 \%)$, les répondants sont peu en accord avec le fait que leurs activités ont un réel impact sur l'attraction.

Le constat est légèrement différent quant aux perceptions relatives à l'impact de leurs activités sur la rétention. Une majorité de répondants sont très en accord $(36,4 \%)$ ou tout à fait en accord $(24,4 \%)$ avec le fait que leurs activités ont un impact positif sur la rétention de travailleurs au Bas-Saint-Laurent. Ce sont les acteurs régionaux qui œuvrent au sein d'organismes dans le domaine du tourisme et de la culture qui ont le sentiment d'avoir un plus grand impact sur la rétention. Toutefois, près du quart des répondants $(23,1 \%)$ sont peu en accord avec l'impact positif des activités de son organisation sur la rétention de travailleurs dans la région.

En contexte de rareté du personnel, l'ensemble des régions périphériques du Québec fait face au même problème en ce qui a trait à l'attraction et à la rétention des travailleurs. Les constats émergeant de ce portrait sont donc capitaux pour les acteurs œuvrant dans ces régions.

La majorité des acteurs régionaux sondés contribue directement ou indirectement à la création et au maintien des emplois existants. Ce 
constat est positif en matière d'attraction et de rétention de la main-d'œuvre, car les perspectives professionnelles pour les travailleurs découlent directement de la disponibilité des emplois. Toutefois, les facteurs liés à la qualité de vie gagneraient à être davantage promus. Bien que l'emploi demeure un facteur central dans la décision de migrer vers une région ou d'y demeurer, les questions liées à la qualité de vie peuvent pallier certains facteurs économiques désavantageux pour un travailleur ${ }^{6}$. Plus particulièrement, les politiques familiales qui incluent l'aide à la recherche d'un emploi pour le conjoint seront salutaires pour attirer et retenir des couples à deux carrières et des familles ${ }^{7}$ Par ailleurs, le parcours de vie des travailleurs à attirer et à retenir est à prendre en considération par les acteurs régionaux dans les actions mises en place. Qu'il s'agisse d'œuvrer en amont auprès des étudiants du secondaire, de rapatrier les jeunes diplômés originaires de la région, d'attirer des familles, des travailleurs expérimentés ou des personnes qui n'ont aucun lien avec la région, les pratiques et les stratégies doivent tenir compte de la réalité de la population ciblée.

La promotion de ces caractéristiques régionales, qu'il soit question d'attraction ou de rétention des travailleurs, passe de plus en plus par le déploiement d'une image positive de la région. Dans le contexte actuel du marché de l'emploi, les employeurs exercent de plus en plus d'efforts afin de diffuser, de développer et de maintenir une image positive, soit une " marque employeur » distinctive. Les régions périphériques du Québec, pour se distinguer les unes des autres, tendent aussi de plus en plus vers cette stratégie pour attirer et retenir les travailleurs. Le déploiement d'une image de marque régionale est un processus où les régions doivent identifier la valeur spécifique qu'elles ont à offrir aux travailleurs potentiels et actuels, communiquer cette proposition de valeur pour attirer et retenir les travailleurs et finalement, soigner cette image en l'intégrant complètement à sa culture régionale afin que la région soit réellement attractive et fidélisante auprès des travailleurs. À la lumière des résultats obtenus, ces stratégies devraient certes s'adresser à la population locale, mais aussi à l'extérieur des frontières des régions périphériques afin de mieux faire connaître cette image positive aux travailleurs d'autres régions. Toutefois, ce processus doit s'harmoniser avec celui déployé par les employeurs de la région afin d'être conséquent auprès des travailleurs potentiels et actuels.

En effet, la concertation entre les acteurs régionaux et les employeurs s'avère certainement un facteur de succès des activités liées à l'attraction et à la rétention des travailleurs. La complexité de ces questions force les collectivités et les entreprises à travailler ensemble pour surmonter les pénuries de main-d'œuvre dans les régions rurales et éloignées des grands centres. Bien que les caractéristiques régionales puissent être mises en évidence par les entreprises ellesmêmes dans leurs pratiques d'attraction et de rétention de la main-d'œuvre, les acteurs régionaux, qui sont bien au fait des attraits régionaux, peuvent jouer un rôle à plus grande échelle. En mettant en place des stratégies concertées et planifiées, ces derniers auront peutêtre davantage le sentiment de faire la différence.

Dans cet ordre d'idées, le soutien aux entreprises de la région, surtout les PME, par les acteurs régionaux dans leurs efforts d'attraction et de rétention des travailleurs devient essentiel. En ce sens, un tel soutien pourrait se traduire par l'offre de formations en gestion des ressources humaines, par du soutien dans la valorisation des caractéristiques régionales (par exemple avec des outils de promotion régionale destinés aux employeurs) et par la création d'un réseau formel de partage d'information entre les employeurs.

Finalement, un autre enjeu lié à l'attraction et à la rétention pour les acteurs régionaux des régions ressources à grands espaces est la disparité des besoins et des réalités entre les MRC d'une même région. La rareté des travailleurs affecte certains territoires alors que d'autres se dévitalisent faute de résidents, souvent au profit des capitales régionales. Les mouvements migratoires se font donc aussi à l'intérieur des frontières de la région. Il convient alors que les actions des acteurs régionaux tiennent compte de cette double réalité dans la mise en action de pratiques d'attraction et de rétention. Il s'agirait donc d'identifier les besoins spécifiques à chacun des territoires pour aligner les interventions sur ces besoins distincts. 
Dans le contexte sociodémographique actuel, la poursuite de la mobilisation régionale autour de la problématique de l'attraction et de la rétention de la main-d'œuvre dans les régions périphériques du Québec s'avère nécessaire pour le développement économique et social de celles-ci, ainsi que pour l'atteinte des objectifs stratégiques des organisations qui y sont situées.

\section{Le déploiement d'une image de marque régionale est un processus où les régions doivent identifier la valeur spécifique qu'elles ont à offrir aux travailleurs potentiels et actuels, communiquer cette proposition de valeur pour attirer et retenir les travailleurs et finalement, soigner cette image en l'intégrant complètement à sa culture régionale.}

\section{BIBLIOGRAPHIE}

${ }^{1}$ Weng, Q. et McElroy, J. C. (2010). « HR environment and regional attraction : An empirical study of industrial clusters in China », Australian Journal of Management, vol. 35, n 3, p. 245-263.

${ }^{2}$ Miles, R. L., Marshall, C., Rolfe, J. et Noonan, S. (2006). " The attraction and retention of professionals to regional areas », Australasian Journal of Regional Studies, vol. 12, $\mathrm{n}^{\circ} 2$, p. 129-152.

${ }^{3}$ Deschênes, A.-A., Beaudry, C., Laflamme, J. et Aguir, M. (2013). « La mesure de l'attraction des travailleurs du savoir dans une région : vérification d'un modèle factoriel de l'attraction régionale ", Actes du Congrès de l'AGRH (Association francophone de gestion des ressources humaines), p. 1-17.

${ }^{4}$ BID.

${ }^{5}$ Yigicanlar, T., Baum, S. et Horton, S. (2007). " Attracting and retaining knowledge workers in knowledge cities », Journal of Knowledge Management, vol. $11, \mathrm{n}^{\circ} 5$, p. 6-17.

${ }^{6}$ Becker, G. S. (1975). Human Capital : Theoretical and Empirical Analysis, with Special Reference to Education, Chicago, University of Chicago Press.

${ }^{7}$ Becker, K., Hyland, P., et Soosay, C. (2013). « Labour attraction and retention in rural and remote Queensland communitites "), Australasian Journal of Regional Studies, vol. $19, \mathrm{n}^{\circ} 3$, p. 342-368.

\section{NOTES}

${ }^{a}$ Andrée-Anne Deschênes est étudiante au doctorat en sciences de l'administration à l'Université Laval.
${ }^{\mathrm{b}}$ Catherine Beaudry est professeure en gestion des ressources humaines à l'Université du Québec à Rimouski.

'Josée Laflamme est professeure en marketing à l'Université du Québec à Rimouski. 